A special objection to alcohol is that it favors coagulation of the blood, leading to embolism and hypostasis - and the still more potent objection, that it increases hyperinosis, which must necessarily develop a higher degree of inflammatory action, involving contiguous tissues in the consuming flame.

If these agents are objectionable because they favor the progress of the disease, a most important question presents itself: What is best calculated to arrest the disease? If a building is on fire the first thing is to destroy the combustibility of the burning material. This principle holds good in inflammation. Subdue as speedily as possible its main supporters. It is admitted that in pneumonitis, either in adults or young children, there is hyperinosis before the pneumonitis is developed, and this constitutes the main factor in the disease. Destroy the excess of fibrine, and you have the disease under control. If there was no hyperinosis in inflammation of the lungs in young children, as some contend, we would only have the mildest form of catarrhal fever. I admit that it is far less than in older children and adults, but there is quite enough to produce the most disastrous results and destroy life. The many forms of embolism, the plugging of the air tubes by coagulating lymph, producing atelectasis, the inflammation resulting from hypostasis, and the extension of the inflammation from the bronchia to the lobules, and from lobule to lobule and from tissue to tissue, are unmistakable evidences of hyperinosis, or excess of fibrine in the blood.

It is admitted by all writers on the subject that the exudate is sufficiently fibrinous to produce an imperfect form of hepatization in most cases. This is another proof of hyperinosis. How to efficiently meet this indication is an important question. The observing housewife long ago discovered that if she had a tough bird to deal with, by applying bicarbonate of soda in liberal quantities at night the bird would be nice and tender for breakfast. The alkali had dissolved the fibrine in the bird, which is just what we want to do in the blood of the pneumonic patient. In mild cases I begin with the benzoate of soda, as a cough syrup. If, however, the case is threatening, I use the more powerful agent, ammonium carbonate, in doses of one to three grains, dissolved in water and syrup, and give every two to four hours. This destroys the tenacious quality of the mucus secreted by the bronchia, and causes it to be thrown off in the usual way, and not to adhere to the walls of the air tubes, interfering with the respiratory functions. This form of medication should be kept up throughout the attack, as it will prevent those dangerous complications to which I have frequently referred in this paper by reducing the excess of fibrine. Another remedy, which accomplishes mechanically the same result in removing the tenacious mucus from the bronchia, is an emetic of sulphate of copper or alum. This is a valuable means of relief, and should not be overlooked.

This ammonium carbonate is not only a powerful defibrinizer, but is equally a decarbonizer, which is effected by its chemical action and the elimination of this poisonous agent through the kidneys and lungs. It is also a safe and very efficient stimulant - far safer and better than alcohol in any form. Another valuable procedure is heat and moisture in the form of a flax-seed or corn-meal poultice, applied to the entire thorax, and kept up with great regularity. This is a thousand times better and safer to relieve cough, pain, and restlessness than opiates.

A remedy that has a tendency to expand the capillary vessels and prevent congestion and gangrene is eucalyptus, a medicine that I have found very useful in pneumonic inflammation of young children. If there is any malarial complication we must not fail to give quinine; and if there are signs of weakening of the heart's action we may give with advantage tincture of digitalis, which is our most reliable heart tonic. If a counter-irritant is needed, we may employ a very mild croton oll liniment, or mustard mixed with the poultices may be used instead.

Mild aperients or enemata may be given as required.

The limited time allowed me forces this paper to be only suggestive, and not by any means exhaustive. If, however, it should encourage a spirit of inquiry, and a more thorough investigation of the pathology and treatment of the inflammatory diseases of the lungs of young children, I will be fully compensated for the labor and thought devoted to its preparation.

\section{THE RADICAL CURE OF CERTAIN FORMS OF HERNIA BY A NEW OPERATION.}

BY REUBEN A. VANCE, M.D., CLEVELAND, O.

Professor of Operative Surgery and Clinical Surgery in the Medical Department of the University of Wooster.

[Read to the Section of the American Medical Association on Surgery and Anatomy.]

The study of nature's method of curing an oblique inguinal hernia, shows there are two important processes at work-one, a band of adventitious tissue about the neck of the sac constantly tending to contract and close the abdominal opening at the inner ring; the other, the return to place of the two layers of transversalis fascia, the separation of which originally permitted the viscera to protrude, and the reunion of which forms a valve strong enough to prevent a recurrence of the hernial protrusion. Both processes must operate if the patient is to be cured. The frequency with which these measures are interfered with by local conditions, explains why so few patients with hernia recover spontaneously.

Trusses, by keeping the viscera in place and allowing the structures about the neck to contract, favor the cure of the lesion. The same is true of certain surgical operations. A moment's consideration of the anatomy of oblique inguinal hernia, and a glance at the pathological processes in operation in such cases, will show why so few are permanently cured, despite the perfection of nature's processes for the accomplishment of that end. The neck of the sac can not contract, for it is almost constantly distended by some part of the abdominal viscera. The displaced folds of transversalis fascia cannot return to their proper position, for the same reason. Not only 
this ; abdominal pressure tends to dilate the hernial orifice, while the protruded viscera force the inner ring downward and towards the middle line. Nevertheless, the surgeon can readily return the hernia, and place the parts in such position that nature can employ her reparative powers to best advantage. Examine an oblique inguinal hernia in the dead body, and it will be found that by proceeding in a certain way the sides of the inner ring-it matters not how largely it may be dilated or how far displaced-can be brought together. To do so, however, traction must be made upon the segment of the circle next the median line, so as to draw it downwards and outwards. 'This proceeding converts the ring into a slit, the long axis of which is directed downward and inward. One or more stitches properly inserted will hold the parts in this position. The anterior and posterior walls of the inguinal canal can be brought in apposition in the same way. Finally, the pillars of the external ring can be transfixed and united by suture. The closure of the internal ring, and its conversion into a simple slit; the union between the anterior and posterior walls of the inguinal canal thus brought about; and finally, the attachment of the pillars of the external ring, combine to transform the former open passage way of the inguinal canal into a firm, unyielding valve, that renders hernial protrusion an impossibility. Imagine these steps taken on the person of one suffering from oblique inguinal hernia. Would not the parts be placed in the best possible condition for such union as would result in a radical cure of the hernia? Experience abundantly attests that such is the fact. That the reparative processes may be understood in their completeness, it must be borne in mind that peritoneum differs from integument or mucous membrane, in that to procure union it suffices to press serous surfaces together, while to unite either of the others the severed edges must be brought together, and retained in apposition until adhesion occurs. Consequently the peritoneal surfaces at the inner ring, in the canal and between the pillars of the inguinal outlet, when apposed in the manner described above, adhere and blend together within a few hours; in time the marks of the oblique slit at the inner ring disappear by the gradual contraction of the band of adventitious tissue that marked the neck of the hernial sac; the formerly separated folds of transversalis fascia are brought together and firmly blended by the same process, while the union of the serous walls of the inguinal canal, and the attachment of the pillars of the external ring, complete the steps that mark the re-establishment of such a valve as alone can make this weak portion of the abdominal wall sufficiently strong to resist the tendency to protrusion of the abdominal contents.

I will mention in this connection that I now have a patient preparing for an operation for the cure of right inguinal hernia, who formerly had double hernia of that character. In this case the left external ring and inguinal canal are still open, protrusion is prevented by contraction of the neck of the old sac, uniting the folds of transversalis fascia. Here we have one of nature's conservative provisions effecting a cure; occasionally this process is prevented by dis- placement of the band of adventitious tissue at the neck, instead of the opening between hernial sac and peritoneal cavity closing and the continuity of the transversalis fascia being re-established, thus curing the hernia, the constriction is forced into the canal, and a partial diaphragm is formed between the neck and fundus of the hernial sac. Although cases similar to the one above alluded to, demonstrate that the transversalis fascia is the main element in preventing hernia, yet experience shows that it is desirable in operating for the cure of rupture to obliterate that portion of the sac in the inguinal canal as well as close the internal and external rings. Devise some plan for carrying the inner portion of the transversalis fascia into contact with the outer, at the internal ring, and the other steps in the attempt to convert the open inguinal canal into a resisting valve are easy of execution. The following method has been very successful in my hands :

Place the patient in a recumbent posture, reduce the hernia by invaginating the scrotum, determine the situation of the internal and external rings and the axis of the inguinal canal. Mark these boundaries on the skin with tincture of iodine, and then draw a short line at right angles to the axis of the inguinal canal, from the upper and inner to the lower and outer border of the internal ring. Next force the unarmed end of a Dowell's hernia needle through skin, fascia, aponeurosis of the external oblique, and tissues intervening between the latter and the parietal layer of the peritoneum, into the abdominal cavity, locating the puncture at the lower and outer end of the short line drawn at right angles to the axis of the inguinal canal.

Carry the end of the needle cautiously upward and inward, in the direction of the just-mentioned short line, until its point is about half an inch beyond the border of the inner ring; then force the point straight through all the structures above it and make it penetrate the skin. The end of the needle heretofore in the surgeon's hand is charged with a strong strand of silk, and in the further manipulation of the instrument great care must be exercised that none of the abdominal viscera be needlessly injured. The threaded end of the needle is passed into and ont of the abdominal cavity, but the instant its point passes beyond the upper and inner border of the sac, its course is changed, and instead of drawing the threaded end out, the latter is forced downward through the tissues of the inguinal canal by the side of, but without the lower and outer border of the internal ring, and then into the same canal in aponeurosis, external oblique, subcutaneous fascia and integument, the needle made when first introduced. Thus the strand of silk enters and leaves by the same canal so far as the structures between the aponeurosis of the external oblique and the skin are concerned, but below that all is different. In effect: a noose is thrown from the fixed layer of transversalis fascia to the movable one, and the latter with its peritoneal envelope and intervening adventitious tissue of the neck of the sac, is drawn downward and outward, and so fastened in its new position as to obliterate the internal ring without injuring the spermatic cord. 
A single suture used in this manner initiates reparative processes that alone suffice to prevent hernial protrusion.

The first suture is introduced, but not tightened; its extremities are left in care of an assistant, and the needle is again threaded for use. Its, insertion for the second suture is peculiar, and is in imitation of Dowell's plan; the integument and all loose subcutaneous tissue are elevated between the thumb and fingers of the right hand, if the patient has left inguinal hernia, the point of the thumb just touching the line marking the axis of the inguinal canal. The needle is grasped in the left hand, and its unarmed end is thrust through the duplication of tissues, its point entering about midway on the line marking the axis of the inguinal canal. As soon as the point appears through the fold of skin it is grasped and the armed end drawn into the tissues. This manouvre carries the end of the needle armed with silk into contact with the aponeurosis of the external oblique; the needle is now so manipulated that this extremity is forced through the aponeurosis into the inguinal canal over the spermatic cord, through the posterior wall of the canal and thence out of the abdominal wall at the point the curvature of the needle causes it to emerge. The unarmed end follows, but is not permitted to come further than the surface of the aponeurosis of the external oblique, the direction of the needle is reversed, its point kept close to the surface, of the aponeurosis and finally brought out at the opening through skin and superficial fascia it made when inserted. 'This makes a noose that can, in oblique hernia, simply bring the posterior wall of the inguinal canal into contact with the anterior, or in cases of long standing in which the internal opening is displaced, attach the margins of the pillars of the external opening, as well as approximate the surfaces of the canal.

A third suture is carried into place in the same manner, but it is inserted into the pillars of the external ring just above the level of the cord, and its extremities emerge from an opening at the lower end of the line marking the axis of the canal. During these manipulations the index finger of the operator invaginates the scrotum and occupies the inguinal canal, in which position it can guide the needle in its passage through the parts about the internal ring and inguinal canal. The sutures passed, they are carefully tightened, beginning with the one first introduced, and the influence of each estimated by the finger in the canal before being finally fastened. A medium sized key separated from the abdominal wall by four folds of antiseptic gauze, should be used to receive the ligature knots. Some point on the ring of the key will do to support the knot on the first suture, the handle of the key will bear the others. The patient had better be anæsthetized with ether for this operation, as the surgeon can then proceed leisurely with his work. After the ligatures are secured the patient should be removed to his bed, and for the next week maintain the recumbent posture. At the end of seven days the bandages are removed and the ligatures taken out. Should any local tenderness develop, the surgeon should at once control it with rubber bags of ice.
I have operated in 32 cases by Dowell's method; in the I9 cases in which I have used the procedure set forth above, the result, so far as the present condition of the patient is concerned, is all that could be desired.

\section{MEDICAL PROGRESS.}

Operations on the Kidneys.--Nephrectomy.Mr. J. Knowsley Thornton gives the record of three successful cases of nephrectomy in the Lancet. The first was published June, r880, but is again given in connection with the others. It was performed on a child of seren, who had suffered with swelling of the abdomen since she was two years old. The diagnosis of renal tumor having been made, an antiseptic aspiration was performed, resulting in the removal of six pints and a half of rather dark and cloudy urine. Two months later the tumor had re-filled and the left kidney was removed through an incision in the median line to the left of the umbilicus. Bichloride of methylene was the anæsthetic used. The ureter was found to be merely a thin fibrous cord. Wound healed by first intention on the sixth day, and the patient was running about the ward on the fourteenth day.

The second case was in a woman of 26 , who, after her third pregnancy, eleven days after labor, suffered from inflammation in the right iliac fossa, followed by pain and swelling in the left leg. Six weeks after delivery pain persisted in the right side, which was spasmodic and along the course of the ureter to the right thigh. A fluctuating tumor of considerable size was found in the right loin, and the urine was loaded with pus, ammoniacal and offensive. The kidney was cut down upon through the loin, and Mr. Thornton notes the much greater amount of hæmorrhage as compared with the abdominal section, and the very imperfect knowledge to be obtained as to the condition of the kidney. A sacculated kidney was found, with very offensive pus; two drainage tubes were introduced; the greater part of the wound closed and antiseptic dressings applied. The second day after the operation the bladder urine was free from albumen. The urine soon showed signs of carbolism, and on the fifth he changed to eucalyptus gauze, but in a very short time pus soaked through sixteen layers of the gauze and teemed with bacteria. Ultimately the wound healed fairly well, but the temperature rose, pain increased, the appetite failed, and thirty-five days after the first operation the kidney was removed through the abdominal walls by an incision along the outer border of the right rectus abdominis. After removal the wound was sponged with tincture of iodine, and as there was still some oozing, perchloride of iron was applied, a drainage tube was used, the wound closed, and the end of the ureter brought outside and fastened with a pin. The kidney weighed I lb. $2 \frac{1}{2} \quad \mathrm{oz}$. The wounds healed completely on the eighteenth day, after which the patient passed from a pint to a pint and a half of healthy urine daily. The constitutional disturbance was decidedly less after the nephrectomy than after the nephrotomy. 